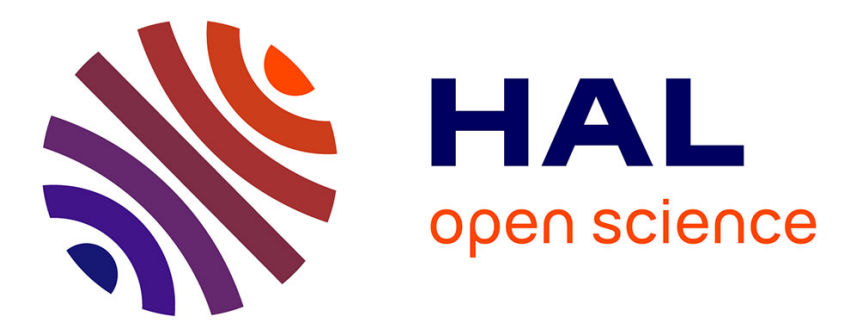

\title{
Les céréales à paille hôtes de Meloidogyne naasi Franklin. II. - Variabilité du comportement multiplicateur ou résistant de variétés cultivées en France
}

\author{
Françoise Person-Dedryver, Danielle Pannetier
}

\section{To cite this version:}

Françoise Person-Dedryver, Danielle Pannetier. Les céréales à paille hôtes de Meloidogyne naasi Franklin. II. - Variabilité du comportement multiplicateur ou résistant de variétés cultivées en France. Agronomie, 1985, 5 (1), pp.55-62. hal-00884733

\section{HAL Id: hal-00884733 https://hal.science/hal-00884733}

Submitted on 1 Jan 1985

HAL is a multi-disciplinary open access archive for the deposit and dissemination of scientific research documents, whether they are published or not. The documents may come from teaching and research institutions in France or abroad, or from public or private research centers.
L'archive ouverte pluridisciplinaire HAL, est destinée au dépôt et à la diffusion de documents scientifiques de niveau recherche, publiés ou non, émanant des établissements d'enseignement et de recherche français ou étrangers, des laboratoires publics ou privés. 


\title{
Les céréales à paille hôtes de Meloidogyne naasi Franklin. II. - Variabilité du comportement multiplicateur ou résistant de variétés cultivées en France
}

Françoise PERSON-DEDRYVER

avec la collaboration technique de Danielle PANNETIER

I.N.R.A., Laboratoire de Zoologie, Centre de Recherches de Rennes, F 35650 Le Rheu

RÉSUME

\begin{abstract}
La prévision des pullulations de Meloidogyne naasi Franklin et des risques de dommages consécutifs à ses attaques nécessite la connaissance de la qualité d'hôte des céréales à l'égard du nématode. Les potentialités d'hôte de variétés d'avoine, d'orge et de blé largement utilisées en France sont estimées dans ce travail.

Les plantes à tester sont cultivées sur sol contaminé à 2 niveaux d'infestation : l'un fort (entre 18 et $26 \mathrm{~L} 2 / \mathrm{g}$ de sol), l'autre faible (entre 3 et $7 \mathrm{~L} 2$ ). Après une extraction en juillet, le comptage des femelles et des œufs révèle une importante variabilité selon les variétés de chaque espèce de céréales étudiée. Néanmoins, le classement de ces espèces suivant leurs potentialités d'hôte vis-à-vis de $M$. naasi est possible.

L'orge d'hiver est, parmi les céréales testées, celle qui fournit le plus de femelles et d'œufs par plante pour les deux potentiels infectieux. Globalement, l'orge de printemps et le blé d'hiver ont des capacités d'hôte identiques, mais bien inférieures à celles de l'orge d'hiver. L'avoine (de type hiver ou printemps) peut être considérée comme un très mauvais hôte dans les conditions de culture françaises.

Dans la pratique agricole, la limitation des populations infestantes dans le sol peut passer par la culture d'avoine ou de variétés de blé d'hiver ou d'orge de printemps multipliant peu le nématode.
\end{abstract}

Mots clés additionnels : Nématode, Avena, Hordeum, Triticum, infestation.

Cereals as hosts of Meloidogyne naasi Franklin. II. - Variability in the susceptible or resistant behaviour of varieties grown in France.

The damage caused by Meloidogyne naasi Franklin in cereal production may be foreseen, if the host behaviour of different cereal species for the nematode is known. With this aim, a study of host quality was made on oat, barley and wheat varieties widely cultivated in France.

All tested plants were grown on infested soil at two degrees of infestation : the first was large and averaged about 18 and $26 \mathrm{~L} 2$ stage larvae per $g$ of soil, and the second was small, between 3 and $7 \mathrm{~L} 2$. Extraction and counting of nematodes issued from cereal roots in July showed wide variability in the production of females and eggs on the varieties of each species. However it was still possible to classify them as hosts.

Winter barley was the cereal species tested which gave the largest number of females or eggs per plant, whatever the infestation level. Generally spring barley and winter wheat were equally good hosts, but much less so than winter barley. Winter or spring oats were very poor hosts under French field conditions. In agricultural practice, the growing of poor hosts such as oats or some varieties of spring barley and winter wheat, should decrease the infestation level of $M$. naasi in the soil and thus provide a method of control.

Additional key words : Nematode, Avena, Hordeum, Triticum, infestation.

\section{INTRODUCTION}

Les potentialités d'hôtes des céréales à paille vis-àvis de Meloidogyne naasi Franklin sont estimées par leur capacité à produire en faible ou forte quantité des femelles et œufs du nématode. La connaissance de cette qualité d'hôte de chaque espèce de céréale donne des éléments indispensables à la prévision des risques d'attaques dommageables sur les cultures qui suivront dans la rotation. Ces risques sont d'autant plus grands que la céréale cultivée fait partie des meilleurs hôtes du nématode. Plusieurs travaux concernent les grami- 
nées, mais peu d'auteurs comparent entre elles les capacités d'hôtes des différentes céréales cultivées. Les études d'AlLEN et al. (1970) aux Etats-Unis signalent que l'orge est un meilleur hôte pour $M$. naasi que l'avoine. Depuis, CoOK et al. (1976) montrent en Grande-Bretagne que les blés de printemps multiplient plus le nématode que l'orge de printemps, l'avoine de type hiver ou printemps étant un hôte très médiocre. Ces résultats sont confirmés par YORK \& GUILE en 1982 et sont comparables à ceux enregistrés par GoORIS \& D'HERDE (1977) en Belgique et par CAUBEL et al. (1971) en France. Après une étude sur 2 variétés, GOORIS \& D'HERDE (1976) constatent que le blé de printemps multiplie plus le nématode que le blé d'hiver. Ce classement des différentes espèces suivant leur qualité d'hôte vis-à-vis de $M$. naasi est établi essentiellement d'après le nombre de galles induites. Seuls GOORIS \& D'HERDE (1977) effectuent une classification plus précise après comptage des œufs et femelles présents sur la totalité des systèmes racinaires des plantes analysées.

Les variétés de blé et d'orge d'hiver qui constituent les principales composantes culturales de la production céréalière française sont peu étudiées hors de France. Les variétés d'orge de type printemps ou d'avoine, dont le comportement vis-à-vis de $M$. naasi est bien connu en Grande-Bretagne et en Belgique, ne sont pas toujours utilisées en France. La connaissance du caractère multiplicateur des différentes espèces et variétés de céréales les plus employées dans nos conditions pédoclimatiques françaises s'avère intéressante. Elle doit être à l'origine de conseils à donner à l'agriculteur français pour éviter des dommages dus à M. naasi.

L'objet de ce travail est l'étude de la qualité d'hôtes vis-à-vis de $M$. naasi de variétés d'avoine, d'orge et de blé largement cultivées en France. L'étude est faite à l'aide de tests réalisés sur sol naturellement infesté, car ce sont les seuls à donner des résultats correspondant à ceux enregistrés dans nos conditions de culture (PERSON-DedRyVER, 1984). Le dénombrement des femelles et œufs sur chaque plante testée permet la détermination de la qualité d'hôte des différentes espèces de céréales ainsi que les limites de sa variation intraspécifique ; cette variabilité correspond aux différences notées entre variétés d'une même espèce.

\section{MATÉRIEL ET MÉTHODES}

\section{A. Matériel}

\section{Le nématode}

Des sols infestés par $M$. naasi sont prélevés durant le mois de septembre précédant les tests, dans des parcelles préalablement cultivées en blé du domaine expérimental de la station INRA du Rheu (Ille-et-Vilaine). Deux niveaux d'infestation sont utilisés pour cette étude : l'un faible, compris entre 3 et 7 larves infectieuses L2 par g de sol et proche du seuil de nuisibilité (PERSON-DEDRYVER, 1983), l'autre plus élevé, situé entre 18 et $26 \mathrm{~L} 2$.

\section{Le végétal}

La plupart des cultivars de blé, d'orge et d'avoine choisis représentent les variétés qui sont les plus multipliées en France au cours de l'année 1980 et qui constituent toujours en 1983 la plus grande partie des emblavures françaises.

Des cultivars d'orge de printemps, «Morocco », «Welam » et $P$ 31-322-1, reconnus pour être résistants au développement d'un autre nématode des cultures céréalières, Heterodera avenae Woll., ont été ajoutés à la liste des variétés testées.

\section{B. Méthodes}

\section{Les tests de la qualité d'hôte des céréales}

La plupart des tests s'effectue sur sol infesté contenu dans des bouteilles en matière plastique d'1,5 1 renversées et conservé en conditions extérieures dès début septembre. Les caryopses sont plantés prégermés. Les autres tests sont réalisés en conditions de plein champ suivant des essais en dispositifs blocs avec des parcelles de $1,3 \times 4,5 \mathrm{~m}$.

Les semis s'effectuent fin octobre ou début novembre pour les variétés de type hiver et en mars ou début avril pour celles de type printemps ; 4 répétitions sont réalisées par variété. Toutes les plantes étudiées une même année de culture sont impérativement examinées en juillet. Les notations sont effectuées sur plantes prélevées suivant une méthode déjà décrite (PERSON-DEDRYVER, 1984) ; elles concernent le poids des systèmes racinaires et les indices pondérés de galles. Les femelles et œufs de $M$. naasi sont extraits des systèmes racinaires après broyage, par la méthode centrifugation flottaison (COOLEN \& D'HERDE, 1972). Leur comptage s'effectue au microscope stéréoscopique.

\section{Analyses statistiques des résultats}

Les différences existant entre les qualités d'hôte des diverses variétés appartenant à une même espèce ou un même type de céréale hiver ou printemps, sont recherchées à l'aide d'analyses de variance. Si nécessaire, un test de DUNCAN, au seuil de risque 5 p. 100 , est réalisé pour classer les moyennes de femelles ou d'œufs formés, ainsi que celles des indices de galles ou de poids de racine. La comparaison globale des taux de multiplication des variétés au niveau interspécifique est faite grâce à des analyses de variance à modèles hiérarchisés à 2 critères de classification (DAGNELIE, 1970). Cette méthode est également utilisée pour juger l'influence des niveaux d'infestation sur les potentialités d'hôte des variétés appartenant à une même espèce.

Pour assurer l'homogénéité des variances, après vérification, il a été nécessaire de transformer en $\log \mathrm{x}+1$ toutes les données relatives aux nombres de femelles et d'œufs.

\section{RÉSULTATS}

L'analyse des résultats concernant le caractère multiplicateur ou résistant des céréales vis-à-vis de $M$. naasi s'effectue, dans un $1^{\text {er }}$ temps, par espèce 
végétale étudiée en différenciant les variétés de type hiver de celles de type printemps. Le facteur important que constitue le potentiel infectieux avant culture $y$ est pris en considération. Dans un $2^{\mathrm{e}}$ temps, nous comparons les qualités d'hôte des différentes céréales testées, en tenant compte de l'aspect variétal et du taux d'infestation avant culture.

\section{A. Etude du caractère hôte des diverses espèces de céréales à paille vis-à-vis de $M$. naasi}

\section{Le blé d'hiver}

Toutes les variétés de blé étudiées multiplient le nématode, mais aucune différence significative concernant la formation des femelles et des œufs ne peut se voir entre cultivars testés en présence d'un fort ou d'un faible potentiel infectieux (tabl. 1).

Pour un taux d'infestation de $20 \mathrm{~L} 2 / \mathrm{g}$ de sol, les variétés «Top » et « Roazon », qui multiplient le plus le nématode, ont des indices pondérés de galles (définis antérieurement, PERSON-DEDRYVER, 1984), significativement supérieurs à ceux de cultivars tels que « Capitole » et " Champlein » qui sont de moins bons hôtes de $M$. naasi (tabl. 1).

Nous avons déjà démontré que des expériences menées, comme c'est ici le cas, 2 années différentes, l'une en pot, l'autre en plein champ, donnent un classement identique des variétés selon leur rang d'hôte (PERSON-DEDRYVER, 1984). D'après cette constatation, nous avons comparé les résultats provenant des 2 séries expérimentales. A faible population infestante, il y a plus d'œufs comptés par femelle qu'à forte population $(F=16,31)$ et les systèmes racinaires des plantes cultivées au champ sont plus petits car difficiles à prélever totalement $(\mathrm{F}=141)$. Par contre, le nombre de femelles formées par plante, les indices de galles sont plus importants à forte qu'à faible infestation avec des $F$ respectivement de 9,81 et 7,56.

\section{L'orge}

\section{a) L'orge de type printemps}

L'étude n'est réalisée qu'à forte infestation. Aucune différence significative n'est notée statistiquement dans la formation des femelles, œufs et galles entre variétés, la variabilité entre plantes étant grande et le nombre de répétitions faible (tabl. 2). Cependant, des écarts non négligeables, concernant les nombres de femelles formées par $\mathrm{g}$ de racine, existent entre les variétés qui multiplient le moins le nématode, telles que «Morocco », et celles qui donnent le plus de femelles, telles que "Cérès".

Pour un cultivar donné, les valeurs extrêmes enregistrées parmi les nombres de femelles formées par $\mathrm{g}$ de racine sont très souvent éloignées l'une de l'autre et sont à l'origine d'écarts-types assez grands.

\section{b) L'orge de type hiver}

Les nombre moyens de femelles formées par $g$ de racine et par variété sont parfois très différents d'un cultivar à un autre, que l'infestation soit faible ou forte (tabl. $3 \& 4$ ), mais les analyses statistiques ne révèlent pas de différences significatives dans la formation de ces femelles, ni dans celle des œufs et des galles.

TABLEAU 1

Formation des femelles, œufs et galles de Meloidogyne naasi sur variétés de blé d'hiver en présence d'un fort ou faible potentiel infectieux. Production of females, eggs and galls of Meloidogyne naasi on winter wheat varieties at high or low infestation level.

\begin{tabular}{|c|c|c|c|c|c|c|c|}
\hline \multirow[t]{2}{*}{$\begin{array}{l}\text { Variétés } \\
\text { blé d'hiver }\end{array}$} & \multirow[t]{2}{*}{$\begin{array}{l}\text { Potentiel } \\
\text { infectieux } \\
\mathrm{L} 2 / \mathrm{g} \text { de sol }\end{array}$} & $\begin{array}{l}\text { Femelles } / g \\
\text { de racine }\end{array}$ & OEufs/femelle & \multirow{2}{*}{$\begin{array}{c}\text { Femelles/ } \\
\text { plante } \\
\text { m }\end{array}$} & \multirow{2}{*}{$\begin{array}{c}\text { OEufs/plante } \\
\text { m }\end{array}$} & \multirow{2}{*}{$\begin{array}{l}\text { Indice } \\
\text { pondéré de } \\
\text { galles } \\
\text { m }\end{array}$} & \multirow{2}{*}{$\begin{array}{c}\text { Poids de } \\
\text { racine/ } \\
\text { plante } \\
\text { m }\end{array}$} \\
\hline & & (p) & (p) & & & & \\
\hline \multicolumn{8}{|c|}{ Essais $1979-1980$ en pot } \\
\hline Capitole & & $64(56-78)$ & $26(15-31)$ & 360 & 9345 & $50^{\mathrm{ab}}$ & 5,54 \\
\hline Castan & & $77(8-174)$ & $19(4-26)$ & 329 & 7280 & $62^{\mathrm{bc}}$ & 4,51 \\
\hline Champlein & 20 & $79(13-100)$ & $31(8-48)$ & 266 & 6860 & $45^{a}$ & 3,88 \\
\hline Caton & & $87(11-154)$ & $44(23-60)$ & 374 & 12068 & $60^{\mathrm{abc}}$ & 4,95 \\
\hline Hardi & & $92(66-130)$ & $39(27-61)$ & 475 & 18252 & $65^{\mathrm{bc}}$ & 5,40 \\
\hline Roazon & & $108(38-170)$ & $18(8-32)$ & 420 & 8323 & $73^{c}$ & 4,10 \\
\hline Top & & $144(44-190)$ & $18(11-26)$ & 949 & 17545 & $72^{c}$ & 6,02 \\
\hline \multicolumn{8}{|l|}{$\begin{array}{l}\text { Essais 1980-1981 } \\
\text { au champ }\end{array}$} \\
\hline Capitole & & $68(22-142)$ & $46(19-88)$ & 96 & 4933 & 38 & 1,62 \\
\hline Arminda & & $70(35-96)$ & $65(25-143)$ & 161 & 7086 & 57 & 2,19 \\
\hline Hardi & 7 & 77 ( 4-162) & $33(10-56)$ & 104 & 3068 & 39 & 1,14 \\
\hline Lutin & & $81(17-184)$ & $54(22-88)$ & 135 & 4578 & 41 & 1,40 \\
\hline Fidel & & $88(12-148)$ & $40(17-79)$ & 243 & 5603 & 57 & 2,22 \\
\hline Roazon & & $105(66-165)$ & $53(33-63)$ & 211 & 10948 & 60 & 2,05 \\
\hline Talent & & $122(73-212)$ & $53(44-62)$ & 290 & 16361 & 58 & 2,15 \\
\hline Rivoli & & $126(40-227)$ & $47(20-83)$ & 183 & 7158 & 47 & 1,33 \\
\hline Top & & $129(69-218)$ & $33(20-52)$ & 329 & 9371 & 54 & 2,60 \\
\hline
\end{tabular}

$\mathrm{m}=$ moyenne sur 4 répétitions ; $(\mathrm{p})=$ valeurs extrêmes minimale ou maximale; test de DuNCAN : les valeurs affectées d'une même lettre $\mathrm{a}, \mathrm{b}$ ou $c$ ne diffèrent pas significativement entre elles au seuil 0,05 . 
TABLEAU 2

Formation des femelles, ceufs et galles de Meloidogyne naasi sur variétés d'orge de printemps en présence d'un fort potentiel infectieux : $21 \mathrm{~L} 2 / \mathrm{g}$ de sol.

Production of females, eggs and galls of Meloidogyne naasi on spring barley varieties at high infestation level : 21 L2 per $g$ of soil.

\begin{tabular}{|c|c|c|c|c|c|c|}
\hline \multirow[t]{2}{*}{$\begin{array}{l}\text { Variétés orge } \\
\text { de printemps }\end{array}$} & $\begin{array}{l}\text { Femelles/g } \\
\text { de racine }\end{array}$ & OEufs/femelle & \multirow{2}{*}{$\begin{array}{c}\text { Femelles/ } \\
\text { plante } \\
\text { m }\end{array}$} & \multirow{2}{*}{$\begin{array}{c}\text { OEufs/plante } \\
\text { m }\end{array}$} & \multirow{2}{*}{$\begin{array}{l}\text { Indice } \\
\text { pondéré } \\
\text { de galles } \\
\text { m }\end{array}$} & \multirow{2}{*}{$\begin{array}{l}\text { Poids } \\
\text { racine/ } \\
\text { plante } \\
\text { m }\end{array}$} \\
\hline & (p) & (p) & & & & \\
\hline \multicolumn{7}{|c|}{ Essais 1980-1981 en pot } \\
\hline Morocco & $32(11-\quad 88)$ & $37(18-54)$ & 72 & 3600 & 70 & 1,88 \\
\hline Menuet & $73(8-192)$ & $44(32-50)$ & 163 & 5548 & 87 & 2,31 \\
\hline P $31-322-1$ & $89(6-243)$ & $84(83-154)$ & 325 & 28649 & 73 & 3,53 \\
\hline Welam & $92(13-199)$ & $79(20-109)$ & 177 & 8829 & 70 & 2,05 \\
\hline Aramir & $98(3-163)$ & $14(6-27)$ & 119 & 1425 & 75 & 1,46 \\
\hline Georgie & $102(10-176)$ & $31(13-57)$ & 127 & 5457 & 80 & 2,34 \\
\hline Cornel & $114(42-204)$ & $15(0-30)$ & 283 & 7296 & 67 & 1,94 \\
\hline Rika & $130(15-403)$ & $37(23-66)$ & 408 & 11281 & 65 & 1,79 \\
\hline Bérac & $165(7-356)$ & $20(11-31)$ & 533 & 11983 & 75 & 2,36 \\
\hline Bétina & $313(49-746)$ & $69(3-228)$ & 500 & 16688 & 65 & 1,87 \\
\hline Cérès & $381(9-1232)$ & $24(0,80-42)$ & 1129 & 45640 & 80 & 1,86 \\
\hline
\end{tabular}

$\mathrm{m}=$ moyenne sur 4 répétitions $;(\mathrm{p})=$ valeurs extrêmes minimale et maximale.

TABLEAU 3

Formation des femelles, œufs et galles de Meloidogyne naasi sur variétés d'orge d'hiver en présence d'un fort potentiel infectieux: $18 \mathrm{~L} 2 / \mathrm{g}$ de sol.

Production of females, eggs and galls of Meloidogyne naasi on winter barley varieties at high infestation level : 18 L2 per $g$ of soil.

\begin{tabular}{|c|c|c|c|c|c|c|c|}
\hline \multirow[t]{2}{*}{$\begin{array}{c}\text { Variétés orge } \\
\text { d'hiver }\end{array}$} & \multicolumn{2}{|c|}{$\begin{array}{l}\text { Femelles/g } \\
\text { de racine }\end{array}$} & OEufs/femelle & \multirow{2}{*}{$\begin{array}{c}\text { Femelles/ } \\
\text { plante } \\
\mathrm{m}\end{array}$} & \multirow{2}{*}{$\begin{array}{c}\text { OEufs/plante } \\
\text { m }\end{array}$} & \multirow{2}{*}{$\begin{array}{c}\text { Indice } \\
\text { pondéré } \\
\text { de galles } \\
\mathrm{m}\end{array}$} & \multirow{2}{*}{$\begin{array}{c}\text { Poids } \\
\text { racine/ } \\
\text { plante } \\
\text { m }\end{array}$} \\
\hline & $\mathbf{m}$ & (p) & (p) & & & & \\
\hline \multicolumn{8}{|c|}{ Essais 1980-1981 en pot } \\
\hline Thibaut & 144 & $\left(\begin{array}{ll}10-335\end{array}\right)$ & $14(6-19)$ & 653 & 11480 & 90 & $5,95^{\mathrm{abc}}$ \\
\hline Ager & 173 & $(0-450)$ & $41 \quad(0-99)$ & 560 & 39871 & 80 & $3,89^{\mathrm{a}}$ \\
\hline Monarque & 209 & $(76-375)$ & $11(2-15)$ & 1270 & 13560 & 95 & $5,54^{\mathrm{ab}}$ \\
\hline Alpha & 250 & $(94-411)$ & $64(11-93)$ & 1490 & 109366 & 93 & $5,97^{a b c}$ \\
\hline Banteng & 266 & $(113-384)$ & $28(4-85)$ & 2173 & 41196 & 100 & $8,62^{b c}$ \\
\hline Nymphe & 278 & $(106-518)$ & $19(8-34)$ & 1537 & 28051 & 90 & $5,94^{\mathrm{abc}}$ \\
\hline Sympa & 281 & $(179-373)$ & $65(35-115)$ & 1345 & 101447 & 90 & $7,49^{a b c}$ \\
\hline Senta & $314^{*}$ & $(157-497)$ & $13 *(2-22)$ & $3042^{*}$ & $41085^{*}$ & $97^{*}$ & $9,72^{\text {bcd }}$ \\
\hline Malta & 325 & $(254-436)$ & $48 \quad(11-79)$ & 1752 & 93243 & 95 & $5,62^{a b c}$ \\
\hline Astrix & 361 & $(150-474)$ & $29(11-52)$ & 2530 & 78949 & 100 & $6,73^{a b c}$ \\
\hline Galion & 373 & $(155-567)$ & $11(8-14)$ & 4008 & 58772 & 100 & $10,36^{\mathrm{cd}}$ \\
\hline Antarès & 395 & $(274-486)$ & $14(8-20)$ & 5369 & 70731 & 100 & $13,61^{\mathrm{d}}$ \\
\hline Monlon & 435 & ( $19-1091)$ & $17(7-34)$ & 2400 & 19506 & 93 & $6,98^{a b c}$ \\
\hline Barberousse & 491 & ( $54-1004)$ & $19(8-35)$ & 3047 & 64832 & 85 & $5,08^{\mathrm{ab}}$ \\
\hline
\end{tabular}

$\mathrm{m}=$ moyenne sur 4 ou $8^{*}$ répétitions $;(p)=$ valeurs extrêmes minimale et maximale ; test de DuNCAN : les valeurs affectées d'une même lettre $a, b, c$ ou $d$ ne diffèrent pas significativement entre elles au seuil 0,05 .

Les nombres de femelles ou d'œufs rapportés à la plante entière dépendent de la variété mais également du poids racinaire. Ainsi, à fort potentiel infectieux, " Antarès ", " Galion " et " Senta ", dont les plantes ont les 3 plus grandes moyennes de poids de racine, font partie des 4 variétés donnant le plus de femelles par plante. Quel que soit le taux d'infestation, " Galion », "Antarès » et « Astrix » sont des variétés très favorables à la multiplication du nématode, alors qu'« Ager " et " Thibaut " sont des hôtes moins efficaces.

La comparaison de toutes les données obtenues dans l'étude des diverses variétés en fonction du degré d'infestation ne montre qu'une seule différence signi- ficative : elle concerne uniquement les femelles développées par $\mathrm{g}$ de racine dont le nombre augmente avec le potentiel infectieux $(F=41,08)$.

\section{L'avoine}

Les avoines cultivées en France sont toutes de mauvais hôtes du nématode (tabl. 5). Seules les variétés "Sirène ", de type printemps, et «Peniarth», de type hiver, permettent le développement de plus d'1 femelle par $g$ de racine à forte infestation. Ces femelles pondent en général très peu d'œufs. Les indices pondérés de galles restent inférieurs à 50 . 
TABLEAU 4

Formation des femelles, oufs et galles de Meloidogyne naasi sur variétés d'orge d'hiver en présence d'un faible potentiel infectieux: $3 \mathrm{~L} 2 / \mathrm{g}$ de sol.

Production of females, eggs and galls of Meloidogyne naasi on winter barley varieties at low infestation level: 3 L2 per $g$ of soil.

\begin{tabular}{|c|c|c|c|c|c|}
\hline $\begin{array}{c}\text { Variétés orge } \\
\text { d'hiver }\end{array}$ & $\begin{array}{l}\text { Femelles/g de racine } \\
\text { m (p) }\end{array}$ & $\begin{array}{l}\text { OEufs/femelle } \\
\text { m (p) }\end{array}$ & $\begin{array}{l}\text { Indice pondéré } \\
\text { de galles } \\
\mathrm{m}\end{array}$ & \multicolumn{2}{|c|}{$\begin{array}{l}\text { Nombre de plantes } \\
\text { ayant un systeme racinaire }\end{array}$} \\
\hline \multicolumn{6}{|l|}{ Essais 1978-1979 en pot } \\
\hline Thibaut & $16(4-24)$ & $18(4-36)$ & 87 & 1 & 3 \\
\hline Sonja & $33(8-52)$ & $22(12-37)$ & 100 & & 4 \\
\hline Ager & $34(8-46)$ & $22(13-36)$ & 100 & & 4 \\
\hline Igri & $38(16-78)$ & $65(15-158)$ & 100 & & 4 \\
\hline Gerbel & $70\left(\begin{array}{ll}16 & -128\end{array}\right)$ & $18(15-23)$ & 100 & & 4 \\
\hline Robur & $87(24-174)$ & $32(7-81)$ & 100 & & 4 \\
\hline Le Peuple & $95(30-160)$ & $24(22-26)$ & 95 & 2 & 2 \\
\hline Malta & $98(15-192)$ & $16(8-25)$ & 100 & & 4 \\
\hline Banteng & $100(10-356)$ & $26(13-50)$ & 90 & 1 & 3 \\
\hline Neger & $140\left(\begin{array}{ll}4 & -438\end{array}\right)$ & $30(19-62)$ & 85 & 1 & 3 \\
\hline Galion & $170(82-298)$ & $20(15-28)$ & 100 & & 4 \\
\hline Astrix & $199(6,6-508)$ & $31(5-62)$ & 80 & 1 & 3 \\
\hline Antarès & $294(16-602)$ & $24(16-34)$ & 100 & & 4 \\
\hline
\end{tabular}

$\mathrm{m}=$ moyenne sur 4 répétitions ; $(\mathrm{p})$ = valeurs extrêmes minimale et maximale.

TABLEAU 5

Formation des femelles, œufs et galles de Meloidogyne naasi sur variétés d'avoine en présence d'un fort potentiel infectieux.

Production of females, eggs and galls of Meloidogyne naasi on oat varieties at high infestation level.

\begin{tabular}{|c|c|c|c|c|c|c|c|c|}
\hline \multirow[t]{2}{*}{$\begin{array}{l}\text { Variétés } \\
\text { avoine }\end{array}$} & \multirow[t]{2}{*}{$\begin{array}{l}\text { Potentiel } \\
\text { infectieux } \\
\mathrm{L} 2 / \mathrm{g} \text { de sol }\end{array}$} & \multicolumn{2}{|c|}{$\begin{array}{l}\text { Femelles/g } \\
\text { de racine }\end{array}$} & OEufs/femelle & \multirow{2}{*}{$\begin{array}{c}\text { Femelles/ } \\
\text { plante } \\
\mathrm{m}\end{array}$} & \multirow{2}{*}{$\begin{array}{c}\text { OEufs/plante } \\
\text { m }\end{array}$} & \multirow{2}{*}{$\begin{array}{l}\text { Indice } \\
\text { pondéré de } \\
\text { galles } \\
\text { m }\end{array}$} & \multirow{2}{*}{$\begin{array}{l}\text { Poids de } \\
\text { racine/ } \\
\text { plante } \\
\text { m }\end{array}$} \\
\hline & & $\mathrm{m}$ & (p) & (p) & & & & \\
\hline \multicolumn{9}{|l|}{ Essais $1979-1980$ en pot } \\
\hline \multicolumn{9}{|l|}{ Printemps } \\
\hline Chérie Noire & & 0 & & 0 & 0 & 0 & 13,33 & 0,77 \\
\hline Cravache & & 0 & & 0 & 0 & 0 & 13,33 & 2,14 \\
\hline Gambo & & 0 & & 0 & 0 & 0 & 17,50 & 2,66 \\
\hline Noire de Moyencourt & & 0 & & 0 & 0 & 0 & 12,50 & 2,07 \\
\hline Rivale & & $0,07(0$ & $-0,2$ & $4 \quad(0-12)$ & 0,18 & 2,2 & 46,67 & 3,94 \\
\hline Maris Tabard & & $0,09(0$ & $-0,27)$ & $2,33(0-7)$ & 0,17 & 1,17 & 20 & 1,18 \\
\hline Leanda & 26 & $0,1 \quad(0$ & $-0,4 j$ & $1,17(0-3,5)$ & 0,27 & 1,25 & 26,67 & 2,33 \\
\hline Manoire & & $0,1 \quad(0$ & $-0,4)$ & $4(0-8)$ & 0,32 & 0,32 & 32,50 & 2,39 \\
\hline Tarra & & $0,13(0$ & $-0,4)$ & $11,5(0-34,5)$ & 0,39 & 13,57 & 36,67 & 2,42 \\
\hline Maris-Oberon & & $0,27(0$ & $-0,8)$ & $0,83(0-2,5)$ & 0,80 & 1,99 & 40 & 3,49 \\
\hline Samba & & $0,4 \quad(0$ & $-0,8)$ & $10,77(0-24)$ & 1,50 & 9,19 & 55 & 3,08 \\
\hline Selma & & $0,45(0$ & $-1,41)$ & $3,67(0-8)$ & 0,55 & 1,89 & 32,50 & 1,78 \\
\hline Margam & & $0,66(0$ & $-1,17)$ & $9,44(0-17)$ & 0,83 & 11,33 & 16,67 & 1,11 \\
\hline Sirène & & $8,20(1,5$ & $7-22,78)$ & $15,49(0-38,85)$ & 17,08 & 92,26 & 22,50 & 1,98 \\
\hline \multicolumn{9}{|l|}{ Essais $1979-1980$ en pot } \\
\hline \multicolumn{9}{|l|}{ Hiver } \\
\hline Crin-Noir & & 0 & & 0 & 0 & 0 & 47,50 & 4,04 \\
\hline Hiver ou Prieuré & 20 & 0 & & 0 & 0 & 0 & 47,50 & 7,99 \\
\hline Amandine & & $0,05(0$ & $-0,2)$ & 0 & 0,34 & 0 & 60 & 7,11 \\
\hline Maris-Quest & & $0,1 \quad 0$ & $-0,2)$ & $(0-1)$ & 0,88 & 0,88 & 47,50 & 8,07 \\
\hline Peniarth & & $1,75(1,2$ & $-2,6)$ & $26,1 \quad(15,61-40,62)$ & 14,68 & 385,65 & 47,50 & 8,50 \\
\hline
\end{tabular}

$\mathrm{m}=$ moyenne sur 4 répétitions ; $(p)=$ valeurs extrêmes minimale ou maximale.

\section{B. Etudes comparatives des qualités d'hôtes des diffé- rentes céréales}

La confrontation des données sur la qualité d'hôte des diverses espèces et types hiver ou printemps de céréales testées (tabl. 1 à 5) avec les résultats prove- nant des analyses de variance à modèles hiérarchisés permettent le classement de ces céréales en fonction de leur caractère hôte (fig. 1). Les avoines de printemps ou d'hiver, très mauvais hôtes de $M$. naasi, ont été exclues délibérément de l'étude statistique qui ne se révèle pas nécessaire pour les dissocier de l'orge et du blé. 


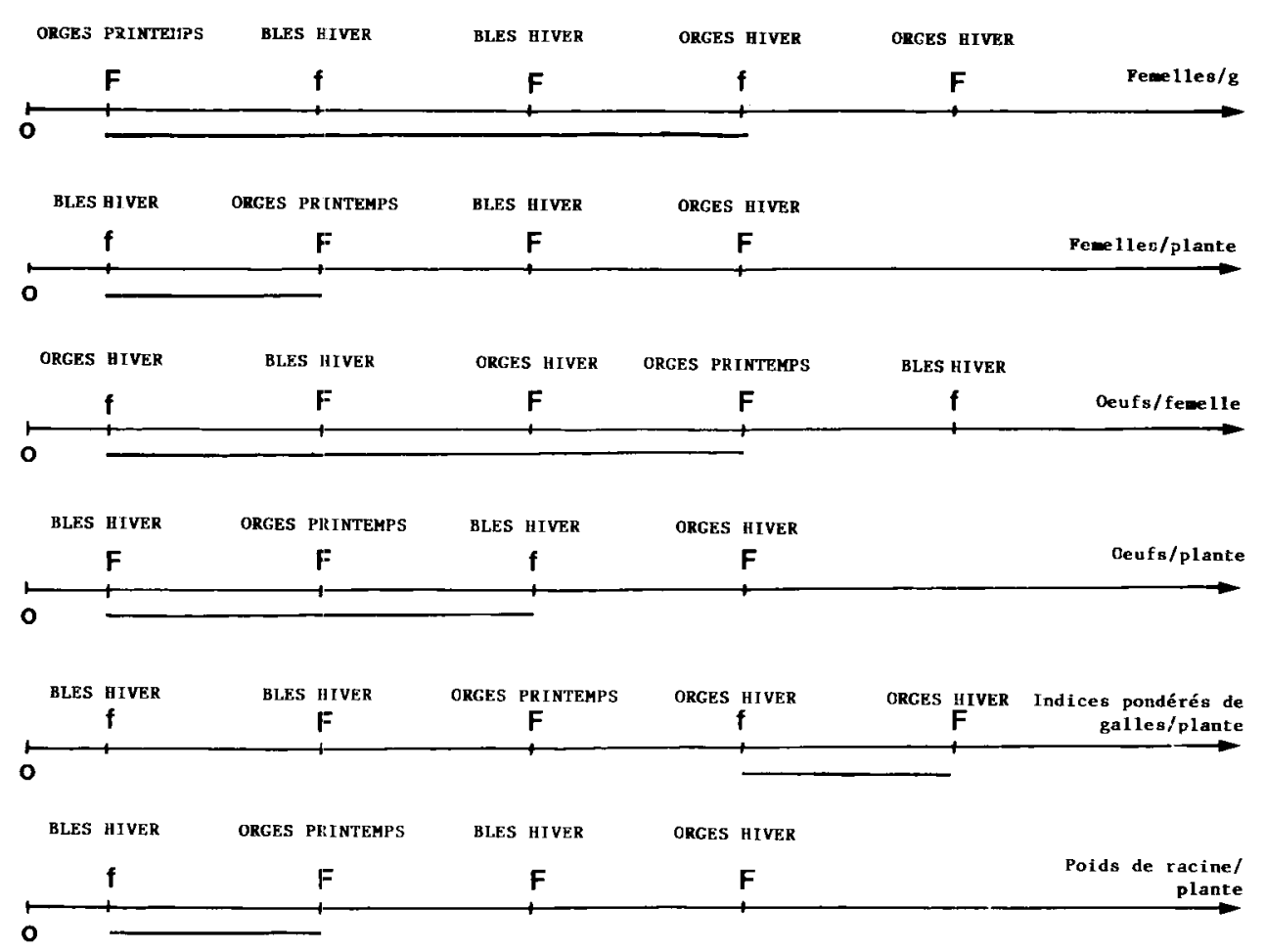

Figure 1

Représentation graphique des différences significatives existant dans la formation des femelles, oufs et galles de Meloidogyne naasi sur orge et blé de type printemps ou hiver; population infestante: forte $F$ ou faible $f$.

- le trait relie des valeurs qui ne sont pas significativement diffé-

\section{La formation des femelles et des cufs}

Quel que soit le potentiel infectieux, l'orge d'hiver reste globalement la céréale testée qui fournit le plus de femelles par $\mathrm{g}$ de racine et par plante et le plus d'œufs par plante. Seul le nombre d'œufs formés par femelle sur orge d'hiver est moins important que celui trouvé sur blé d'hiver cultivé à faible infestation (fig. 1). L'augmentation du potentiel infectieux ne fait qu'accroître les différences notées entre l'orge d'hiver et les autres espèces ou types de céréales. Ainsi, en cas de forte infestation, l'orge d'hiver, "Thibaut», qui multiplie le moins $M$. naasi, donne autant de femelles et d'œufs que "Top », meilleur hôte parmi les blés d'hiver (tabl. 1 \& 3).

A fort potentiel infectieux, certaines variétés d'orge de printemps, telles que "Cérès ", "Bétina " et "Bérac ", multiplient plus le nématode que les cultivars de blé d'hiver étudiés (tabl. 1 \& 2). A l'inverse, la variété "Morocco " est un moins bon hôte que les blés. Globalement, nous ne constatons pas de différence significative dans la formation des femelles par $\mathrm{g}$ de racine et dans celle des œufs par femelle entre les blés d'hiver et l'orge de printemps. Cependant, nous obtenons significativement plus de femelles et d'œufs par plante chez le blé d'hiver (fig. 1).

\section{La formation des galles}

Les indices pondérés de galles décroissent significativement dans l'ordre suivant : orges d'hiver cultivées en cas de forte ou faible infestation, orges de printemps, blés d'hiver à fort puis à faible potentiel infectieux (fig. 1). rentes, après études effectuées par analyse de variance à modèles hiérarchisés.

Diagrams showing significant differences in the production of females, eggs and galls of Meloidogyne naasi on spring or winter barley and wheat.

\section{Le poids des racines}

Les poids racinaires des orges de printemps plantées en pot et des blés d'hiver cultivés uniquement au champ ne diffèrent pas entre eux. Ils sont cependant plus faibles que ceux des blés et orges d'hiver semés en pot, dont les racines sont totalement récupérées. Les orges d'hiver ont les poids racinaires les plus importants (fig. 1).

\section{DISCUSSION - CONCLUSIONS}

\section{A. Les qualités d'hôtes intraspécifiques des céréales}

L'avoine de type hiver ou printemps est, en France et en Belgique (GOORIS \& D'HERDE, 1977), un mauvais hôte du nématode. Cependant, la formation de quelques femelles n'est pas exclue sur un certain nombre de variétés. Dans nos expériences réalisées en conditions extérieures, nous ne pouvons imputer le développement de ces femelles sur avoines à des hautes températures, comme l'ont constaté GoORIS \& D’HERDE (1977) en serre. Seul le facteur variétal est ici responsable. Nos résultats correspondent d'ailleurs tout à fait à ceux trouvés en Europe. Ainsi, la variété de printemps "Selma ", étudiée dans 3 expériences réalisées avec des taux d'infestation supérieurs à $15 \mathrm{~L} 2$ par $\mathrm{g}$ de sol, en Grande-Bretagne (COOK, 1972), en Belgique (GoORIS \& D'HERDE, 1977) et en France, donne respectivement $0,5,1$ et 0,45 femelles formées par $\mathrm{g}$ de racine. "Peniarth " est l'une des variétés d'avoine d'hiver qui, en France et en Angleterre, 
donne le plus de femelles avec respectivement 1,75 ou 1,80 femelles par $\mathrm{g}$ de racine. Nos résultats sur les avoines ne peuvent pas être comparés avec les données américaines obtenues en serre (ALLEN et al., 1970 ; SIDDIQUI \& TAYLOR, 1970), d'autant plus que la classification des variétés est réalisée sur le seul critère de présence ou absence de femelles ou d'œufs sans précisions chiffrées.

Les variétés de printemps ou d'hiver d'orge et de blé étudiées et cultivées en France, sont toutes de bons hôtes pour $M$. naasi, comme d'autres cultivars testés par OGUNFOWORA en Angleterre (1977), GOORIS \& D'HERDE en Belgique (1976 \& 1977), CAUBEL et al. en France (1971). Des différences dans la potentialité d'hôte existent cependant entre les variétés appartenant à une même espèce ou au même groupe de céréales semées en hiver ou au printemps. Mais nous n'avons jamais pu les mettre en évidence statistiquement, car les variances sur les moyennes de femelles ou d'œufs formés sont très grandes et sont inhérentes aux tests utilisés ainsi qu'au nombre réduit de répétitions vu l'ampleur du travail à réaliser. Nous pouvons cependant différencier les variétés d'une même espèce en fonction de leur qualité d'hôte, puisque nous obtenons des classements identiques, que les tests soient réalisés sur une ou plusieurs années (PERSONDEDRYVER, 1984).

Ainsi, « Monlon », « Barberousse », « Antarès », "Astrix » et «Galion » sont les variétés d'orge d'hiver qui développent le plus le nématode. "Cérès » et «Bétina » sont les meilleurs hôtes parmi les orges de printemps et «Top » reste le blé d'hiver qui multiplie le plus $M$. naasi.

\section{B. Différences interspécifiques de la qualité d'hôte des céréales}

Comme CoOK et al. (1976) en Grande-Bretagne, nous constatons que, dans nos conditions de culture, l'orge de printemps multiplie plus $M$. naasi que l'avoine de type hiver ou printemps.

Nos travaux démontrent que l'orge d'hiver est incontestablement la céréale qui donne le plus de femelles et d'œufs, quel que soit le potentiel infectieux. Globalement, l'orge de printemps et le blé d'hiver ont des capacités identiques en ce qui concerne la formation des femelles par $g$ de racine et d'œufs par femelle. Par contre, le blé d'hiver fournit significativement plus de femelles et d'œufs par plante que l'orge de printemps. Ce résultat s'explique aisément car le poids racinaire des céréales, lié au tallage (MoUle, 1971) est toujours plus important chez les variétés de type hiver que sur celles de type printemps.

La qualité d'hôte du blé d'hiver paraît cependant très limitée puisque le nombre de femelles formées par $\mathrm{g}$ de racine reste stable et statistiquement identique lorsque le potentiel infectieux croît. Tout se passe comme si les larves L2, ayant pénétré en plus grand nombre, déterminent un indice de galles significativement supérieur et se retrouvent en compétition. Il s'ensuit une formation maximum dans le nombre de femelles comptées par g de racine et une ponte d'œufs inférieure par femelle (tabl. 1).

Ces résultats sont d'un grand intérêt pour la pratique agricole, $M$. naasi étant préjudiciable aux cultures de céréales (PERSON-Dedryver, 1983). Pour exploiter, parce qu'elles sont économiquement rentables, des rotations céréalières intensives sur sol infesté, il est préférable de faire intervenir des avoines hôtes médiocres du nématode. Ces avoines limiteront les populations dans le sol, comme peut le faire le maïs (Person-Dedryver, 1983) et les risques de dommages sur les cultures suivantes seront réduits. Pour des raisons économiques, cette céréale est cependant de moins en moins utilisée et l'emploi d'autres espèces doit être envisagé.

En cas de faible infestation, proche de $5 \mathrm{~L} 2$ par $\mathrm{g}$ de sol et du seuil de nuisibilité (PERSON-DEDRYVER, 1983), les variétés d'orge d'hiver multipliant peu le nématode, telles que «Thibaut», «Sonja », "Ager » ou " Igri ", sont à préférer aux cultivars de blé d'hiver ou d'orge excellents hôtes de $M$. naasi, tels que « Galion », «Astrix », "Antarès », « Cérès » ou «Bétina ». En présence de fortes infestations, supérieures à 15 L2 par g de sol, ce sont surtout les blés d'hiver qui limiteront les multiplications du déprédateur, avec cependant le risque de subir des attaques dommageables (PERSON-DEDRYVER, 1983).

Reçu le 2 avril 1984. Accepté le 3 août 1984.

\section{RÉFÉRENCES BIBLIOGRAPHIQUES}

Allen M. W., Hart W. H., Baghott K. V., 1970. Crop rotation controls barley root-knot nematode at Tulelake. Calif. Agric., 24, 4-5.

Caubel G., Lemaire J. M., Rivoal R., 1971. Observations préliminaires sur l'attaque de diverses céréales par Meloidogyne naasi en présence d'Heterodera avenae et d'Ophiobolus graminis. Conférence sur les Nématodes du genre Meloidogyne. O.E.P.P., Antibes, 27-29 octobre $1971,10 \mathrm{p}$.

Cook R., 1972. Reaction of some oat cultivars to Meloidogyne naasi. Plant Pathol., 21, 41-43.
Cook R., York P. A., Chew B. H., 1976. Variation in host reaction to the cereal root-knot nematode Meloidogyne naasi and new host records. Rep. Welsh Plant Breed. Stn. for 1976, 71-72.

Coolen W. A., D'Herde C. J., 1972. A method for the quantitative extraction of nematodes from plant tissue. Publ. Gov. Res. Stn. Nematol. Entomol. Merelbecke, Belgium, 1-77.

Dagnelie P., 1970. Théorie et méthodes statistiques. Applications agronomiques. II. - Les méthodes de l'inférence statistique. Les Presses agronomiques de Gembloux A, S, B, L. Editions J. Duculot S. A., Gembloux, 451 p. 
Gooris J., D'Herde C. J., 1976. Situation hivernale de Meloidogyne naasi Franklin et lutte par rotation culturale. Bull. O.E.P.P., 6, 289-296.

Gooris J., D'Herde C. J., 1977. Study on the biology of Meloidogyne naasi Franklin 1965. Stn. Nematol. Entomol. Res. Merelbecke, Belgique, $165 \mathrm{p}$.

Moule C., 1971. Phytotechnie spéciale. II. Céréales. La Maison rustique, Paris, $236 \mathrm{p}$.

Ogunfowora A. O., 1977. Reaction of barley to cereal root-knot nematode, Meloidogyne naasi. Plant Pathol., 26, 161-166.

Person-Dedryver F., 1983. Meloidogyne naasi : nématode à galles des céréales. Journées d'études "Rotations céréalières intensives », O.N.I.C., 16-17 mai 1983, Paris, 9 p.
Person-Dedryver F., 1984. Les céréales à paille hôtes de Meloidogyne naasi. I. Mise au point de méthodes et résultats préliminaires d'évaluation de la résistance ou du caractère multiplicateur. Agronomie, 4 (10).

Siddiqui I. A., Taylor D. P., 1970. Symptoms and varietal reaction of oats to the Illinois isolate of the barley root-knot nematode Meloidogyne naasi. Plant Dis. Rep., 54, 972-975.

York P. A., Guile C. T., 1982. Effects of previous cropping on yield of spring barley on Meloidogyne naasi infested sites. Rep. Welsh Plant Breed. Stn. for 1982, 111-112. 